Conclusion Assessment of SpA remodelling may not require quantitative assessment since semi-quantitative scoring correlates well with quantified VSMC in partially remodelled SpA. This scoring technique provides an approach to assessment of uterine SpA remodelling in pregnancy pathology.

\section{PM.27 GESTATIONAL DIABETES: IS IT SAFE NOT TO INDUCE?}

doi:10.1136/archdischild-2013-303966.110

K O'Shea, A Makris, S Hamilton, S Pathak. Hinchingbrooke Health Care NHS Trust, Huntingdon, UK

Background Despite recent advances in the management of Gestational Diabetes (GDM), there is a paucity of evidence addressing the ideal timing of delivery in women who are well controlled. If recent proposed changes to diagnostic criteria were to be adopted ${ }^{1}$, the incidence of GDM would increase up to $16 \%$. This could potentially increase induction and caesarean section rate.

Objective To assess the safety of not routinely inducing well controlled gestational diabetic women at 38 weeks.

Methods Retrospective study in a district general hospital.

Outcomes Incidence of fetal macrosomia, stillbirth, caesarean section, shoulder dystocia, third degree tear, postpartum haemorrhage $(\mathrm{PPH})$ and admission to SCBU in this population.

Results In 2012, 157 women were diagnosed as GDM according to WHO/NICE criteria; $47 \%$ were treated with Metformin and $15 \%$ with Insulin. 48 women, well-controlled on diet, Metformin, and/ or Insulin, were allowed to go into spontaneous labour. 12 of these women were eventually induced for post-maturity.

The incidence of macrosomia, emergency caesarean section, third-degree tear, PPH, SCBU admission was 4\%, 12.5\%, 4\%, 4\% and $4 \%$ respectively. There were no cases of shoulder dystocia or stillbirth. These figures are well below the national average.

Conclusion Treating well-controlled gestational diabetics conservatively at term is a safe management option.

\section{REFERENCE}

1. Metzger BE, Gabbe SG, Persson B, Buchanan TA, Catalano PA, Damm P, Dyer AR, Leiva A, Hod M, Kitzmiler JL, et al, International association of diabetes and pregnancy study groups recommendations on the diagnosis and classification of hyperglycemia in pregnancy. Diabetes Care 2010;33:676-682.

\section{PM.28 AUDIT ON CARE AND OUTCOME OF PREGNANCY IN WOMEN WITH MORBID OBESITY}

doi:10.1136/archdischild-2013-303966.111

D Kapoor, J Davison, S Rajendran. Royal Derby Hospital, Derby, UK

Background Obesity is one of the biggest challenges facing maternity services today. Women with obesity have significant higher complication rates and these can potentially be reduced with good quality clinical care.

Objectives To compare current practise at Royal Derby Hospital with the RCOG/CEMACE guideline on management of pregnant women with morbid obesity.

Methods A retrospective case-note audit of all women delivered at $\mathrm{RDH}$ between 01/09/2011 and 31/08/2012 with booking BMI $\geq 40$. Results There were 6252 deliveries at RDH with 140(2.24\%) patients with $\mathrm{BMI} \geq 40$. We analysed 134 case notes. Glucose tolerance test was performed in $96.2 \%$ of patients at 28 weeks gestation and $55.2 \%$ had an anaesthetic review. $47.1 \%$ of women laboured spontaneously, $36.5 \%$ of labours were induced and $16.4 \%$ had elective caesarean section (CS). $67.8 \%$ of women had a normal vaginal delivery, the instrumental delivery and Emergency CS rate were $9.0 \%$ and $23.2 \%$ respectively. $89.6 \%$ of operative deliveries were either performed or supervised by ST6 and above. $96.2 \%$ had active management of $3^{\text {rd }}$ stage and major postpartum haemorrhage occurred in $8.2 \%$ of women. For term deliveries the birth weights ranged from $2620 \mathrm{~g}$ to $5080 \mathrm{~g}$ (21.6\% greater than $4000 \mathrm{~g}) .47 .3 \%$ had postnatal thromboprophylaxis with $81.4 \%$ on sufficient thromboprophylaxis for weight.

Conclusion The audit demonstrated that the guidelines were not being adhered to, particularly for anaesthetic assessment and venous thromboembolism risk. Review of our local guideline with more training for healthcare professionals involved in the care of this group of high risk patients is required to improve maternal and neonatal outcomes.

\section{REFERENCE}

CMACE/RCOG Joint Guideline on 'Management of Women with Obesity in Pregnancy' - March 2010

\section{PM.29 COMPARISON OF HEPATIC PERFUSION BETWEEN PRE-ECLAMPSIA AND NORMAL PREGNANCY}

doi:10.1136/archdischild-2013-303966.112

\section{A Anbazhagan, S Ong. Royal Jubilee Maternity Hospital, Belfast, UK}

Objective We wanted to provide evidence for or against the thus far, unaccepted theory that the genesis of pre-eclampsia arises from the maternal venous circulation. We also wanted to assess hepatic perfusion in pre-eclampsia using 3 dimensional ultrasound.

Methods We measured hepatic portal vein flow in 12 women with normal pregnancy and 11 women with pre-eclampsia using standard Doppler ultrasound. We evaluated the three dimensional (3D) indices of hepatic perfusion: flow index (FI), vascular index (VI) and vascularisation flow index (VFI) which are believed to reflect vascularity and flow intensity. Because of small numbers, a non-parametric test was used to test differences between groups.

Results Hepatic portal vein flow was not different between women with normal pregnancy compared to women with pre-eclampsia [228.1 (215.5-270.6) vs. 283.0 (145.9-344.6); $p=0.90]$. The 3D indices of hepatic perfusion were as follows [FI; 36.3(30.7-42.5) vs. 39.7 (27.7-44.2), $\mathrm{p}=1.00$; VI; 11.7 (3.6-21.2) vs. 3.0 (0.5-7.6), $\mathrm{p}=0.04$; VFI; $4.7(1.2-8.3)$ vs. $1.2(0.1-3.2), p=0.06$ respectively].

Conclusion We were not able to provide evidence in support of the suggestion that the genesis of pre-eclampsia arises from the maternal venous circulation. Because of the wide variability of our data, overall we conclude that there is no difference in the $3 \mathrm{D}$ indices of hepatic perfusion in women with pre-eclampsia compared to normal pregnant women.

\section{PM.30 WITHDRAWN BY AUTHOR}

\section{PM.31 A LITERATURE REVIEW OF INTERFERON BETA TREATMENT IN MULTIPLE SCLEROSIS PATIENTS DURING PREGNANCY - IMPLICATIONS FOR FUTURE RESEARCH}

doi:10.1136/archdischild-2013-303966.113

\section{SK Carney, Y Igzeer. Pennine Acute Trust, Oldham, UK}

Multiple sclerosis is a neurological disease caused by discrete plaques of demyelination at sites throughout the central nervous system caused by a T-Cell mediated immune response with an unknown trigger. It has a lifetime risk of 1:1000 in the UK, and is twice as prevalent in females with the typical onset being between 20 and 40 years of age, namely the childbearing ages.

There are many disease-modifying therapies used to treat Multiple Sclerosis. However, immunomodulatory and immunosuppressive drugs used at any stage of pregnancy may affect fetus formation and/or development. 
Interferons are a group of naturally occurring macromolecules with antiviral, antiproliferative and immunomodulatory properties, and interferon beta is currently the most widely used therapy for multiple sclerosis. However, limited data in primates suggests that interferon beta may be abortifacient. Due to this and due to lack of experience with drug safety, it is usually suggested that either treatment is suspended when a pregnancy is planned, or a critical assessment of the pros and cons of ceasing therapy should be performed.

A literature review of the last ten years identified nine papers, which looked at maternal (relapse rates, mode of delivery) and fetal/ neonatal outcomes (spontaneous abortion, pre-term delivery, birth weight, birth defects, still births and developmental milestones) associated with its use in pregnancy.

The literature review highlighted conflicting results, however, on the whole, for most outcomes most studies did not associate IFN beta use during pregnancy with adverse outcomes. Further trials investigating important maternal, fetal and neonatal outcomes are called for.

\section{PM.32 PLATELET FUNCTION IS SIGNIFICANTLY REDUCED IN THE FIRST TRIMESTER OF PREGNANCY COMPARED TO THE NON-PREGNANT STATE}

doi:10.1136/archdischild-2013-303966.114

${ }^{1} \mathrm{~N}$ Burke, ${ }^{1} \mathrm{~K}$ Flood, ${ }^{1} \mathrm{~A}$ Murray, ${ }^{1} \mathrm{M}$ Dempsey, ${ }^{3} \mathrm{P}$ Dicker, ${ }^{1} \mathrm{M}$ Geary, ${ }^{2} \mathrm{D}$ Kenny, ${ }^{1} \mathrm{~F}$ Malone. 'Obstetrics and Gynaecology, Royal College of Surgeons in Ireland, Rotunda Hospital, Dublin, Ireland; 'Molecular and Cellular Therapeutics, Royal College of Surgeons in Ireland, Dublin, Ireland; '3Epidemiology, Royal College of Surgeons in Ireland, Dublin, Ireland

Abnormalities of platelet function have been implicated in a number of obstetric complications and anti platelet therapy is used to prevent certain conditions. Research of platelet function in pregnancy has yielded conflicting results. We sought to critically evaluate platelet reactivity in pregnancy using an assay which allowed several agonists of varying concentrations to be assessed concurrently and aimed to clarify platelet reactivity in normal pregnancy.

A prospective longitudinal study was performed throughout uncomplicated singleton pregnancies with patients recruited prior to 15 weeks' gestation. They were controlled for a number of factors known to affect platelet reactivity. Blood samples were obtained in each trimester $(n=36)$. Thirty non-pregnant healthy female volunteers also had a platelet assay performed. A modification of standard light transmission aggregometry was used to assess platelet reactivity, with light absorbance measured following addition of 5 different agonists at sub-maximal concentrations. Dose-response curves were plotted and the Ec50 was calculated for each agonist.

Platelet reactivity, as demonstrated by the Ec50, was significantly reduced in the $1^{\text {st }}$ and $2^{\text {nd }}$ trimester of pregnancy compared to the non pregnant state particularly with respect to collagen, $(p=0.002)$. Within the pregnancy cohort the platelet reactivity increased as the pregnancy progressed, most evident in response to arachidonic acid (AA) $(p=0.033)$

This study demonstrates that platelet reactivity is altered in pregnancy, highlighted by the significant reduction in reactivity seen in the $1^{\text {st }}$ trimester. This information will be critically important for designing and interpreting interventions to prevent obstetric complications, such as preeclampsia.

\section{PM.33 THE OBSTETRIC MANAGEMENT OF HAEMOPHILIA CARRIERS AND PATIENTS WITH VON WILLEBRAND'S DISEASE IN LEEDS}

doi:10.1136/archdischild-2013-303966.115

'E Jones, 'R Al-biatty, 'ㄹ Ciantar. 'University of Leeds, Leeds, UK; ' 2 Leeds Teaching Hospitals NHS Trust, Leeds, UK
Background Haemophilia and von Willebrand's disease (vWD) are inherited bleeding disorders that present significant obstetric challenges, including risks of neonatal intracranial haemorrhage and postpartum haemorrhage (PPH). The ideal mode of delivery can also be controversial.

Aims To assess the obstetric management of haemophilia carriers and vWD patients within the Leeds Teaching Hospitals Trust and to identify any maternal/neonatal complications.

Method 10 year retrospective audit of 24 women (10 haemophilia carriers; $14 \mathrm{vWD}$ patients) identified from the Obstetric-Haematology Clinic between 2001 and 2011 in Leeds. Maternal and neonatal management was compared to the BJH guidelines. ${ }^{1,2}$

Results From the 10 haemophilia patients, 9 had antenatal gender identification (7 were male and 5 affected). There were 4 PPHs in the vWD group (not exceeding $800 \mathrm{mls}$ ). Amongst the haemophilia patients 9 had a normal delivery and 1 had an elective C-section. In the vWD group 11 had normal deliveries, 2 had elective C-sections and 1 had a rotational forceps. 1 fetal blood sample was performed in the haemophilia group and 2 fetal scalp electrodes were used in the vWD group (both contraindicated). There was no neonatal morbidity amongst the haemophiliac patients but 4 babies in the vWD group sustained bruising/prolonged bleeding which were forceps and im vitamin K-related respectively.

Conclusions Vaginal delivery was the preferred mode of delivery and was not associated with any significant maternal or neonatal morbidity. Managing these patients through a multidisciplinary approach optimises their antenatal care and ensures that an intrapartum management plan is discussed and clearly documented.

\section{REFERENCES}

1. Lee C, Chi C, Povard $\mathrm{S}$ et al, The obstetric and gynaecological management of women with inherited bleeding disorders - review with guidelines produced by the taskforce of UK Haemophilia Centre Doctors Organisation. Haempohilia 2006;12:301-36.

2. Calmers $E$, Williams $M$, Brennand J et al, Guideline on the management of haemophilia in the fetus and neonate. British Journal of Haematology 2011;154:208-15.

\section{PM.34 IMMUNE THROMBOCYTOPENIA IN PREGNANCY}

doi:10.1136/archdischild-2013-303966.116

JL Hogan, C Walsh, S Higgins, K Murphy, F McAuliffe. National Maternity Hospital, Dublin, Ireland

Immune thrombocytopenia (ITP) is the most common cause of thrombocytopenia in the first half of pregnancy and occurs in one or two per thousand pregnancies. The management of these pregnancies is often directed at maintaining a sufficient platelet count for delivery and other labour ward procedures. A retrospective review, of pregnancies complicated by ITP, was performed to determine mode of delivery and mean platelet counts during pregnancy.

Patients with ITP were identified from the maternal medicine database. Delivery demographics for these patients were obtained from the hospital's database. Platelet counts were obtained for each trimester of pregnancy for the mother and the neonate.

There were 39 pregnancies, complicated by ITP, identified from 2005-2012. 15 were nulliparous and 5 of the patients had two pregnancies during the study period. The majority had a vaginal delivery $(76.9 \%)$. The mean platelet count in the first trimester was $119,200 / \mu \mathrm{l}$ (range $27,000-365,000 / \mu \mathrm{l}$ ). In the second trimester, the mean platelet count fell to $99,400 / \mu \mathrm{l}(16,000-255,000 / \mu \mathrm{l})$ and to $89,000 / \mu \mathrm{l}(22,000-231,000 / \mu)$ in the third trimester. There were 8 patients with platelet counts less than $50,000 / \mu 1$ in the third trimester. The mean neonatal platelet count on day one of life was $200,700 / \mu \mathrm{l}(42,000-414,000 / \mu \mathrm{l})$ and $149,500 / \mu \mathrm{l}(15,000-279,000 / \mu \mathrm{l})$ on day four of life. There were 5 neonates with platelets less than $50,000 / \mu 1$ 